\title{
Aicardi syndrome: a case report
}

\author{
Jean Carlos de Oliveira Menezes 1 \\ Francisco Elvis Farias da Silva 2 \\ Érica Galdino Félix 3 \\ João Carlos Alchieri 4 \\ Joelma Gomes da Silva 5
}

1-3,5 Departamento de Fisioterapia. Faculdade do Vale do Jaguaribe. Av. Nossa Senhora Perpétuo Socorro, 1302. Mossoró, RN, Brasil. CEP: 59.615-050. E-mail: fisiojoelmagomes@gmail.com

4 Universidade Federal do Rio Grande do Norte. Natal, RN, Brasil.

\begin{abstract}
Introduction: the Aicardi syndrome (SA) is characterized as a rare syndrome identified in the presence of three classic characteristics: corpus callosum agenesis, chorioretinal lacunaeand infantile spasms.

Description: data collection involved information reported by the mother and the accompanying physiotherapist describing the patient's clinical history andmajor complications according to clinical evolution, treatment, and therapeutic response. At two months of age, the child presented a delayed neuropsychomotor development and infantile spasms. However, the diagnosis of the syndrome was only performed at six months of life, involving brain magnetic resonance imaging where corneal body agenesis was observed. A multidisciplinary treatment was assembledwith a neuropediatrician, a physiotherapist, a psychologist, a nutritionistand a speech therapist, besides drug treatment with baclofen and phenobarbital.

Discussion: through the established treatment, the child displayedmotor gain, cervical control, improvement of the respiratory condition, and no need forhospital admissions; these outcomescharacterizea good clinical evolution associated with the physiotherapeutic intervention focused on prevention and minimization of respiratory alterationsthatare frequently associated with morbidity and mortality in these cases. The results obtained point out the fundamental role of multidisciplinary intervention in coping with this condition.

Key words Aicardi's syndrome, Agenesis of the corpus callosum, Infantile spasms,
\end{abstract} Chorioretinal lacunae

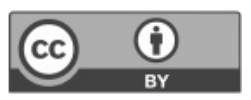




\section{Introduction}

Throughout life, human beings undergo changes that promote their development; a process where the acquisition of a great amount of physical, cognitive, sensorial, emotional, and social abilities take place. ${ }^{1}$

However, several factors may jeopardize the normal course of this process such as environmental or biological conditions and neural, motor, and cognitive disorders leading to the onset of syndromes, ${ }^{2}$ among whichis Aicardi Syndrome (AS).

First identified by French neurologist Jean Aicardi in 1965, AS is diagnosed in the presence of three classic characteristics: agenesis or dysgenesis of corpus callosum, chorioretinal lacunae, and infantile spasms (convulsive crises), or in the presence of two of the classic characteristics accompanied by at least two other major characteristics: vertebral, spinal, or rib malformations, muscular hypotonia, intellectual retardation, or delay in postural acquisition. 3,4

AS is typically a unique case in families and theprobability of a mother having a second child with the same condition is less than $1 \% .5$

Although not yet fully established, it is believed that this pathology is linked to analteration in the $\mathrm{X}$ chromosome, a condition that affects almost exclusively female individuals. 5

Two situations can be presented when it affects males: patient presents a karyotype with 47 chromosomes, that is, 47XXY (a chromosomal anomaly also associated with the Klinefelter Syndrome), or still has 46 chromosomes, however, in a situation of lethality. 5

In the great majority of cases of dizygotic twin sisters, AS affects only one sister, excluding the hypothesis of etiology in the intrauterine environment, infectious causes, or the presence of a teratogenic agent. ${ }^{6}$

More than 4,000 cases are known in the world, and the syndrome'sincidence is estimated between 1 : 105,000 born children. In the United States, this estimate is 1: 167,000 and in Europe between 1: 93,000 and1: 99,000. The mean survival age, even in the most affected individuals, is 16 years (it was only six years oldin the past). The pro-bability of an individual with AS surviving up to 27 years of age is $0.62 \%$; 49 years of age is the longest reported lifetime of an individual with a mild form of the syndrome. 5

Due to the degenerative nature of neuromuscular disorders, there is no current effective treatment besides palliative and preventive treatments about the onset of symptoms. As a rule, the majority of patients require antiepileptics, such as vigabatrin, for adequate seizure control. However, the effect is not as expected inall patients, which makes this alternative treatment limited and with very varied results. ${ }^{7}$

To meet the needs and alleviate their difficulties, patients are submitted to rehabilitation interventions conducted by multidisciplinary teams. A pediatric neurologist specialized in the management of childhood spasms and epilepsy is of great relevance in seizure control in addition to Speech Therapy, Occupational Therapy and Physiotherapy, which should begin as early as possible.?

Nevertheless, even with the support of this team, the need for greater knowledge within the area of this pathologyis evident. The information about this condition is scarce due to its low incidence, which also hinders diagnosis.

Hence, the present study aimed at presenting a case report of a child with Aicardi's syndrome.

\section{Description}

The studied child is female, born at term (40 weeks) and delivered through cesarean; the mother's age was 39 years. The birth weight was $3,760 \mathrm{~g}$, height of $50 \mathrm{~cm}$, cephalic perimeter of $35 \mathrm{~cm}$, APGAR of $8 / 10$, with evidenceof crying and sucking at birth, and without intercurrences in the immediate postpartum period.

At the age of two months, the first symptoms were observed, including delayed neuropsychomotor development and infantile spasms. The patient presented delayed psychomotor development in the functional kinetic evaluation performed at two months and 14 days of life; this outcome was characterized by cervical domain deficit and absence of spontaneous movement with hyporreflexibility of primitive reflexes.

In the supine posture, the upper limbs were lightly flexed at the level of the elbows and in external rotation in the lower limbs, with a normal range of motion. The respiratory evaluation showed the presence of vesicular murmur and adventitious sounds of the snoring type, with no need for ventilatory support.

Given these symptoms, physiotherapeutic interventions were performed daily for 60 minutes each time and aimed at preventing musculoskeletal and respiratory complications.

At six months of age, hospitalization was registered with in a period of 27 days due to respiratory complications, requiring non-invasive mechanical ventilation (NIV). The child presented global hypotonia after this hospitalization. 
Corpus callosum agenesis was observed in the magnetic resonance imaging of the brain performed at six months of age (Figures 1 and 2) justifying the diagnostic hypothesis of Aicardi Syndrome. Multidisciplinary management was started with a neuro-pediatrician, a physiotherapist, and a psychologist every six months. A monthly treatment is maintained with both nutritionist and speech therapist, as well as intervention with a physiotherapist five times a week, in the municipality where the child lives. The management of seizures is conductedusing phenobarbital; baclofen is used for the treatment of spasticity.

Eye examinations performed at 15 months of age included the Eye Movement Exam (EME) in which the visual function indicated the ability to follow light and objects of contrast; the Biomicroscopy exam (BCE) showed transparent cornea and crystalline, and the Fundoscopy Examination (FE) show edretina with normal staining. Based on these exams, it was concluded that the patient did not present chorioretinal lacunae.

The physiotherapeutic intervention started at two months of age, involving daily appointments of respiratory and motor physiotherapy. The patient arrived at the physiotherapeutic care without a definite clinical diagnosis, presenting delayed motor development, lack of cervical control, and no ability to locate or follow sounds.

Sound and visual stimuli evaluations were performed in the first trimester associated with trunk dissociation and alternate reach; sound and visual stimuli interventions soughtafternormalization of tone and inhibition of pathological patterns.
Exercises such as a game of hands and feet, ability to feel the head and hands, and to roll overwere performed seeking to increase body awareness through sensorimotor stimulation and prevention of deformities.

Through these exercises, the child, at almost six months of age, began to bring limbs to the body midline. At the age of nine months, she began to show active but discreet movements in the cervical region, especially in rotation, began holding instruments such as baby bottles and pacifiers, and usingher hands to play with her feet.

This evolution accompanied the physiotherapeutic intervention involving passive stretching, joint mobilization, trunk work with the use of adapted toys, stimulation of cervical and midline control, sedestation training, functional stimulation (stimulus to roll over and pick up objects, and visual stimulation with technological resources and with dolls).

No significant gains were observed in functional independence because the child cannot sit without support, bear the weight of herbody on lower limbs, or walkeven with assistance. She does not speak and respondsonly with smiles to tactile and auditory stimuli. Such dysfunctions prevent from participating in activities of daily living (ADL).

However, despite the limitations observed at three years of age, the child does not present hypotonia, has cervical control, light passive trunk control; can support the headin supine, perform a total roll over, move her legs, display palmar grip and glabellar reflex, and follows objects and sounds.

The performance of respiratory physiotherapy

Figure 1

Normal corpus callosum in a newborn (A) and in a nine-year-old child (B).

A

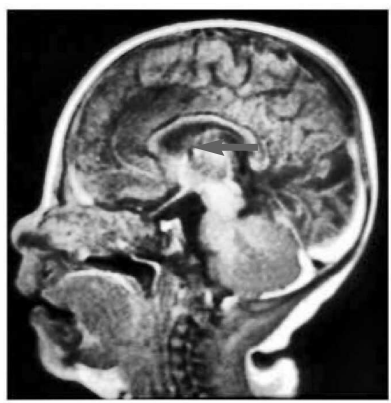

B

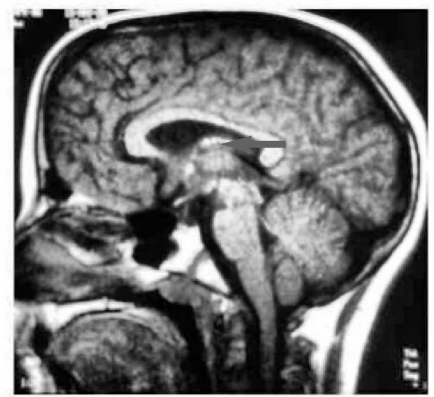

Source: www.pediatricneuro.com 
Magnetic resonance imaging showing corpus callosum agenesis.

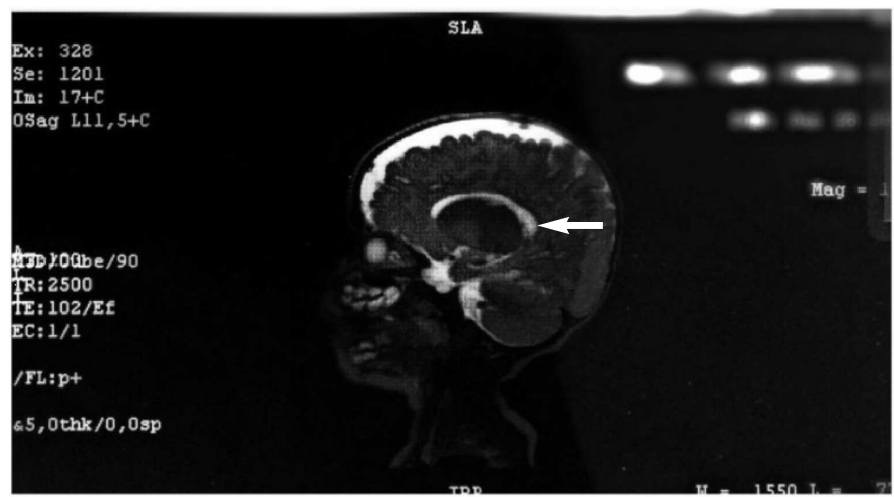

Source: family medical records.

consisted of bronchial hygiene techniques such as pulmonary percussions, compression/decompression, vibrocompressionand postural drainage in order to displace secretion because the child could not expel it voluntarily. The therapeutic approach implemented aimed at improving thoracic pulmonary expandability and oxygenation. This treatment had a significant impact on the patient's overall condition, who no longer required hospitalizations for respiratory infection.

\section{Discussion}

One of the earliest manifestations in the AS diagnosis is infant spasms, which appears early and usually around the third month of life, displayed with rapid muscular contractions and hyperextension of the upper limbs and trunkwith several episodes during the day 3 and in the form of spasms without total seizure control.8,9 The possibility of controlling these spams, although rare, has already been reported in the literature 4 in two Indian children who achieved $100 \%$ control of seizures. This agrees with the present study because the child in this case report does not present any crisis of spasms for two years and four months and is under the possibility of medication suspension.

Corpus callosum agenesis may occur completely or partially, and all children diagnosed with this syndrome have presented this abnormality. $4,8,9$ This diagnosis can be performed even in the gestational period through ultrasonography and indicates suspicion of Aicardi syndrome. 5 However, in the present case, the diagnosis was made through an MRI after the child's birth.

Another AS characteristic isthe presence of chorioretinal lacunae, present in $50 \%$ of the cases, and all children with Aicardi's syndrome reported in the consulted literature.4,8,9 The child in the present study, however, did not present chorioretinal lacunae. All ophthalmologic examinations confirmed retinal, cornea, and crystalline preservation. It is worth noting that the absence of this characteristic does not interfere in the diagnosis, which is based on the presence of two classic characteristics, agenesis of the corpus callosum and infantile spasms along with three main characteristics: muscular hypotonia, intellectual retardation, and delay in postural acquisitions. 3,4

Among the symptoms, a bad spine formation mainly in the thoracic region is a common radiological finding in AS and found in $75 \%$ of affected children. These dysfunctions include congenital fusion of the vertebral bodies, hemivertebrae, and butterfly vertebrae, 10 which was not evidenced in the studied case.

Faced with all symptom characteristics of this syndrome, most of the cases present severe disturbances in psychomotor development, as mentioned in the literature, ${ }^{8}$ with no improvement in neuropsychomotor development delay even with stimulation and physiotherapeutic intervention.

However, this did not occur in the present study since the child responded satisfactorily to the physiotherapeutic treatment and presented cervical control, light passive trunk control, ability to support the head in supine, the ability for total roll over and to accompany objects and sounds, palmar grip, and 
glabellar reflex.

Thus, the observed evolution of the respiratory and motor clinical frame can be related to the implemented physiotherapeutic interventions. This fact fosters the important contribution of the physiotherapist in the multidisciplinary team, necessary for adequate management of AS.

Hence, the importance of bronchial hygiene care is emphasized because the AS survival is only up to $40 \%$ in adolescence and very rare in adulthood; the main complications that lead todeath are related to respiratory problems, mainly due hypersecretion. $4,8,9$

The child, in this case, presented secretion and adventitious sounds, however, with the physiotherapeutic treatment, she did not require hospitalization or hospital care.

The description of this AS case reaffirms the importance of a multidisciplinary team, not only in the patient care, but also in family, as a support to manage the changes in routine and amelioration of signs and symptoms, which allows for an improved prognosis.

\section{References}

1. Oliveira AS. Caracterização do desenvolvimento motor de lactentes de mães adolescentes. Fisioter Pesq. 2013; 20: 349-54.

2. Formiga CKMR, Linhares MBM. Motor development curve from 0 to 12 months in infants Born preterm. Acta Pediatr. 2011; 100: 379-84.

3. Aicardi J. Aicardi syndrome. Brain Dev. 2005; 27 (3): 16471.

4. Banerjee TK, Chattopadhyay A, Manglik AK, Ghosh B. Aicardi syndrome: a reportoffive a Indian cases. Neurol (India). 2006; 54 (1): 91-3.

5. Kroner BL, Preiss LR, Ardini MA, Gaillard WD. New incidence, prevalence, and survival of Aicardi syndrome from 408 cases. J Child Neurol. 2008; 23 (5): 531-5.

6. Velarde JA, Gonzo C, Garcia-Muñoz S, Gil R, Muñoz L. Heterogeneidad clinica e prognostica em el síndrome de Aicardi: a propósito de dos casos. Rev Neural. 1999; 28 (8): $784-5$.
7. Chau V, Karvelas G, Jacob P, Carmant L. Early treatment of Aicardi syndrome with vigabatrin can improve outcome. Neurology. 2004; 63: 1756-7.

8. Aguiar MFM, Cavalcanti M, Barbosa H, Vilela SL, Mendonça JL, Horta E. Síndrome de Aicardi e papiloma do plexo coróide: uma associação rara: relato de caso. Arq Neuropsiquiatr.1996; 54 (2): 313-7.

9. Costa T, Greer W, Rysiecki G, Buncic JR, Ray PN Monozygotic twins discordant for Aicardi syndrome. J Med Genet. 1997; 34 (8): 688 - 91.

10. Baierl P, Markl A, Thelen M, Laub MC. MR imaging in Aicardi syndrome. AJNR.1988; 9: 805-6.

Received on July 21, 2017

Final version presented on August 31, 2018

Approved on September 21, 2018 